\title{
Pain Perception and Effectiveness of the Eutectic Mixture of Local Anesthetics in Children Undergoing Venipuncture
}

\author{
ISABELLE ROBIEUX, CHRISOULA ELIOPOULOS, PAUL HWANG, MARK GREENBERG, \\ VICTOR BLANCHETTE, NANCY OLIVIERI, NAOMI KLEIN, AND GIDEON KOREN \\ Divisions of Clinical Pharmacology [I.R., C.E., N.K., G.K.], Neurology [P.H.] and Hematology-Oncology [M.G., \\ V.B., N.O.J, Department of Pediatrics, Pharmacology and Pharmacy, University of Toronto, and the Research \\ Institute, the Hospital for Sick Children, Toronto, Canada
}

\begin{abstract}
The emulsion of lidocaine and prilocaine (EMLA) is effective in preventing the pain of venipuncture in children. It is therefore important to identify children who could benefit the most from EMLA. We studied the safety and feasibility of two methods of application of EMLA (patch and cream) in a randomized, open-label trial of EMLA patch versus EMLA cream in 160 children with chronic diseases undergoing venipuncture. EMLA patch or cream was applied 60 to 120 min before puncture. Pain was assessed by the children using a visual analogue score. Children also scored the pain of their last puncture and the pain of removing the tape. EMLA patch and cream had similar efficacy (visual analogue scores for the venipuncture were $8.5 \pm 16$ and $9.5 \pm 17$ out of 100 , respectively). Side effects occurred in similar frequencies in the two groups. Adhesiveness of the patch was less effective than that of the cream with Tegaderm. Age was a major determinant of pain perception; younger children recalled more severe pain in their previous puncture. Children recently diagnosed had higher visual analogue scores than those with a long history of chronic disease. We concluded that EMLA patch and cream have a similar efficacy in children undergoing venipuncture. Young children recently diagnosed with chronic disease are most likely to benefit from EMLA. (Pediatr Res 32: 520-523, 1992)
\end{abstract}

\section{Abbreviations}

EMLA, eutectic mixture of local anesthetics VAS, visual analogue score

EMLA is an effective method of local anesthesia for venipuncture, obviating the need for infiltrating local anesthesia by injection. Optimal skin anesthesia is achieved after application of EMLA cream for $60 \mathrm{~min}$ and is maintained up to $2-3 \mathrm{~h} \mathrm{(1)}$. The maximal depth of analgesia is obtained after $120 \mathrm{~min}$ of application and may reach $5 \mathrm{~mm}$ (Bjerring P, Arendt-Nielsen L, manuscript in preparation). Although originally tested in adults, EMLA's efficacy and safety in children undergoing venipuncture was subsequently documented (Bjerring P, Arendt-Nielsen L, manuscript in preparation). The efficacy of EMLA in alleviating pain of venipuncture in children and infants has been recently demonstrated by several groups (3-5). EMLA administration

Received October 9, 1991; accepted June 11, 1992.

Correspondence: Gideon Koren, M.D., Division of Clinical Pharmacology, The Hospital for Sick Children, 555 University Ave., Toronto, Ontario, Canada M5G1X8.

Supported by ASTRA Pharmaceutical, Inc., Etobicoke, Ontario, Canada. may be of particular importance for children with chronic illnesses, who require repeated venipunctures.

We hypothesized that several factors may play a role in perception and recall of needle pain by children: sex, age, factors that may determine sensitization to the painful procedures (6), frequency of venipunctures, and the time since diagnosis of the chronic disease. It was deemed important to identify a subgroup of children for whom anticipation of pain is high and who may therefore benefit more than others from EMLA.

A potential drawback to the use of EMLA is the necessity to apply it at least $60 \mathrm{~min}$ before the puncture. In a previous study, we have shown that in some pediatric outpatient clinics EMLA could be applied without major disruption of the routine schedule. In other clinics, however, the application of EMLA by the parent (or patient) $1 \mathrm{~h}$ before the appointment may be necessary to avoid any disruption or prolonged waiting time (7). The formulation of EMLA as a single unit package (the "EMLA patch") may simplify the application of EMLA by the family.

The objectives of the present study were to compare the feasibility and efficacy of two forms of EMLA and to examine various factors that may determine the perception of pain as reported by children and the effectiveness of skin anesthesia in alleviating the pain associated with venipuncture. In particular, we hoped to identify those children who tend to perceive more pain and who may benefit more from EMLA. Because of the substantial cost of EMLA, hospitals and other medical institutions may need to prioritize who should receive the eutectic mixture and for what indications.

\section{PATIENTS AND METHODS}

The study protocol was approved by our Human Experimentation Review Committee. It was designed as a randomized, open-label trial of EMLA patch versus EMLA cream stratified in four outpatient clinics at the Hospital for Sick Children in Toronto.

Children ranging from 5 to 18 y of age were included after demonstrating a good understanding of the VAS (8). Understanding of the VAS was verified by asking the children to grade levels of pain caused by a mosquito bite, falling on the snow, falling on a road, and slamming a finger in a door. Children with a history of allergy to local anesthetics or those who received a premedication were excluded. Informed, written consent of a parent and assent of the child were obtained before participation.

EMLA is a water emulsion of $25 \mathrm{mg} / \mathrm{g}$ lidocaine and $25 \mathrm{mg} / \mathrm{g}$ prilocaine. Children were randomized to receive either EMLA cream [ $2.5 \mathrm{~g}$ of EMLA cream covered by an adhesive, occlusive tape (Tegaderm)] or an EMLA patch (a single unit dose package of $1 \mathrm{~g}$ of EMLA, using foam medical tape as an adhesive). Both forms were applied 1 to $2 \mathrm{~h}$ before the puncture. 
At the time of the venipuncture, the adhesiveness of the tape and local reactions were recorded. The puncture was performed by a nurse according to the standard procedures of our hospital. After the puncture, the child scored on a VAS the pain of removing the adhesive tape and the pain of the puncture at the site of EMLA application. On this scale, zero denotes no pain and 100 denotes the worst possible pain. In addition, the children were asked to recall, by means of the same VAS, the pain of their last puncture (without EMLA). Children were also asked whether they would like EMLA for their next puncture. Parents and nurses filled out a short questionnaire, as presented in Tables 1 and 2.

Statistical analysis. We used the $t$ test for unpaired data to compare the two treatment groups with respect to pain scores, age, time since last puncture, and time of application of the drug in both males and females. Because most VAS were normally distributed, the $t$ test was deemed appropriate in most cases. However, for data with low baseline values and large variation, such as slight pain associated with patch removal or pain with EMLA use, the nonparametric Mann-Whitney $U$ test was also used. The $\chi^{2}$ test was used to compare the proportion of adverse reactions and incomplete affixment between the two treatment groups.

Analysis of variance for repeated measures was used to compare the three pain scores (pain of last puncture, pain of removing the patch, and pain of puncture after using EMLA). Analysis of variance (factorial analysis) was used to compare pain scores according to race and clinic type. Stepwise regression analysis was used to define correlation between pain scores and age, corrected time since diagnosis, and time since last puncture. Unless mentioned otherwise, data are presented as mean \pm 1 SD.

Sample size was calculated to be able to show significant differences of five points on the VAS scale ranging from 0 to 100 between EMLA cream and patch. We assumed a standard deviation of 12.5 and chose $\alpha=0.05$ and $\beta=0.20$. The large number of patients $(n=160)$ was sufficient to allow stepwise regression with three independent variables with the above power.

\section{RESULTS}

We studied 160 children undergoing venipuncture attending four clinics (Table 3) at the Hospital for Sick Children in Toronto. In the Hematology/Oncology Clinic, enrolled patients had the diagnoses of thalassemia $(n=10)$, sickle cell disease $(n=3)$, leukemia $(n=11)$, hemophilia $(n=8)$, solid tumors $(n=4)$, and other $(n=4)$. Fifteen patients had the puncture for insertion

Table 1. Parents' evaluation of EMLA

1. Do you think the pain your child received from the needle puncture today was less than previous needle punctures?

$$
\text { Less } 87.5 \% \quad \text { Same } 11 \% \text { Worse } 1.5 \%
$$

2. Was the 1-h application time difficult to fit into your schedule? No $94 \%$ Yes $6 \%$

3. Do you think the use of EMLA helped your child cope with his/her illness?

No $37 \%$

Yes $63 \%$

4. Would you want your child to receive EMLA on his/her next visit? No $22 \%$ Yes $78 \%$

Table 2. Nurse's evaluation of EMLA

1. Do you think EMLA was useful in alleviating the pain from the needle?

$$
\text { No } 92 \% \quad \text { Yes } 8 \%
$$

2. Was the use of EMLA disruptive to your routine?

$$
\text { No } 89 \% \quad \text { Yes } 11 \%
$$

3. Do you think the benefit of EMLA outweighs the disruption of your routine?

No $10 \% \quad$ Yes $90 \%$

of an i.v. line for chemotherapy, 12 for transfusion, and 13 for blood work only. The patient populations in the other clinics were more homogeneous. In the Neurology Clinic, all but one patient were epileptics requiring blood work for therapeutic drug monitoring. In the Rheumatology Clinic, patients had diagnoses of lupus, juvenile arthritis, and spondylitis, and blood work was performed as a part of the regular checkup. In the fourth clinic all patients had cystic fibrosis and were examined during their annual or biannual checkup. In each clinic, 20 patients randomly received an EMLA patch and 20 received EMLA cream with Tegaderm.

Comparability of treatment groups. Children in the two treatments groups had similar age and sex distribution as well as similar time intervals since the diagnosis of their disease; time since last puncture was shorter in the patch group $(5 \pm 1 \mathrm{mo})$ than in the Tegaderm group $(3.9 \pm 1 \mathrm{mo})(p=0.02$ in unpaired $t$ test). However, when asked to recall the pain of the last venipuncture, the mean VAS were not statistically different (51 \pm 30 versus $53 \pm 31$ ) (Table 4$)$. Mean time of EMLA application was identical in the two groups $(70 \pm 20 \mathrm{~min})$.

Comparison of adhesiveness. At the time of removal, the patch was incompletely affixed to the skin more often than the Tegaderm [14 (18\%) and five (6\%) cases, respectively $(p=0.026)]$. The pain from removing the adhesive, assessed by the child using a VAS, was similar with patch $(19 \pm 26)$ and Tegaderm $(19 \pm$ 21) (Table 4).

Local adverse reactions. The most common local reaction was pallor at the site of EMLA application and redness at the site of tape (Table 5). Most reactions were mild, and their frequencies were not statistically different between the groups. In two cases $(1.5 \%)$, more serious and prolonged local reactions occurred. A 7 -y-old boy with cystic fibrosis had an itchy rash for $2 \mathrm{~d}$ at both the sites of the cream and the Tegaderm. A 16-y-old girl with rheumatoid arthritis experienced severe pain upon removal of the patch, followed by a painful and itchy rash for $2 \mathrm{~d}$. Another child had a large hematoma at the site of puncture unrelated to the use of EMLA.

Analgesic efficacy of EMLA. Children treated with either patch or cream reported similar levels of pain from the venipuncture $(8.5 \pm 16$ and $9.5 \pm 17$, respectively) (Table 4). Similar results were obtained after exclusion of patients with incomplete affixion $(8.1 \pm 17.0$ and $9.5 \pm 16.9)$. The VAS for the pain associated with the present venipuncture (with EMLA) was significantly less than the pain reported for the last puncture and also less than the pain of removing the tape $(p<0.001)$. In the group studied in the Hematology/Oncology Clinic, the pain score was higher and more variable $(16.6 \pm 24)$ than in the other three clinics (Table $4 ; p<0.01$ ). The major determinant of this variability appears to be the needle size; children requiring blood transfusion $(n=12)$ were treated with a 21 -gauge needle and reported more pain $(35.5 \pm 25)$ than the 27 children in the same clinic pricked with a smaller needle ( 23 gauge) for chemotherapy or blood sampling (VAS $=7.0 \pm 1.1 ; p=0.0002$ ).

Evaluation of EMLA by children. Eighty percent of the children stated that they would wish to receive EMLA for their next puncture; $13 \%$ did not want to and $7 \%$ were not sure. This distribution was similar in all four clinics. Children who said they wanted EMLA for their next puncture were younger $(9.5 \pm$ 3.4 versus $12.5 \pm 2.9 \mathrm{y} ; p=0.01)$ and recalled more pain from the previous puncture $(58 \pm 28$ versus $25 \pm 28 ; p=0.001)$ than children refusing EMLA for subsequent punctures.

Evaluation of pain of last puncture. Because pain scores were identical with both methods of administering EMLA, the data were combined for subsequent analyses. Sex, racial background, and clinic type did not contribute to recall of pain. The age and the time since diagnosis were strongly intercorrelated $(p=0.001)$. To determine whether time since diagnosis (Td) influenced the perception of pain independently from age, we corrected this value for age (cTd) by using the formula $\mathrm{cTd}=($ age-Td)/age, which is the fraction of life free of disease. 
Table 3. Population characterics*

\begin{tabular}{|c|c|c|c|c|c|}
\hline & $\begin{array}{c}\text { Clinic } 1 \\
\text { Hem/Onc }\end{array}$ & $\begin{array}{c}\text { Clinic } 2 \\
\text { Neurology }\end{array}$ & $\begin{array}{c}\text { Clinic } 3 \\
\text { Rheumatology }\end{array}$ & $\begin{array}{c}\text { Clinic } 4 \\
\text { Cystic fibrosis }\end{array}$ & Total (range) \\
\hline$n$ & 40 & 40 & 41 & 40 & 161 \\
\hline Males (\%) & 65 & 55 & 40 & 62.5 & $55(89 \%)$ \\
\hline Age $(y)$ & $10.5 \pm 3.4$ & $11.5 \pm 3.6$ & $11.8 \pm 4.3$ & $10.4 \pm 4.2$ & $11 \pm 3.9(5-18)$ \\
\hline Weight (kg) & $37.5 \pm 17.8$ & $47.7 \pm 19.4$ & $43.2 \pm 16.3$ & $35.9 \pm 15.6$ & $41 \pm 18(15-96)$ \\
\hline Skin allergy $(\%)$ & 7.5 & 0 & 2.5 & 0 & 2.5 \\
\hline Time since diagnosis $(y)$ & $6.0 \pm 4.5$ & $4.9 \pm 3.6$ & $3.6 \pm 4.3$ & $7.8 \pm 4.2$ & $5.6 \pm 4.4(0-22)$ \\
\hline Visits/y & $13.5 \pm 12.9$ & $3.8 \pm 4.1$ & $4.4 \pm 4.4$ & $3.8 \pm 0.7$ & $6.3 \pm 8.1(0-60)$ \\
\hline Time since last puncture (d) & $84.8 \pm 132.4$ & $138.1 \pm 90.5$ & $135.3 \pm 119.1$ & $180 \pm 50$ & $135 \pm 107(1-540)$ \\
\hline
\end{tabular}

* Values are mean $\pm \mathrm{SD}$.

Table 4. Children's pain assessment $(V A S)^{*}$

\begin{tabular}{|c|c|c|c|c|c|}
\hline & Clinic 1 & Clinic 2 & Clinic 3 & Clinic 4 & Total \\
\hline \multicolumn{6}{|l|}{ Patch } \\
\hline Last puncture & $39.8 \pm 31.6$ & $54.9 \pm 25.6$ & $55.5 \pm 33.5$ & $52 \pm 26$ & $51 \pm 29$ \\
\hline Removal of patch & $14.1 \pm 25.0$ & $31.0 \pm 29.5$ & $15.9 \pm 24.5$ & $17 \pm 22$ & $19.5 \pm 26$ \\
\hline Today's puncture & $18.7 \pm 26.7$ & $7.2 \pm 13.1$ & $4.2 \pm 6.3$ & $3.7 \pm 6.4$ & $8.5 \pm 16$ \\
\hline \multicolumn{6}{|l|}{ Cream } \\
\hline Last puncture & $54.9 \pm 28.1$ & $50.5 \pm 33.9$ & $48.8 \pm 31.7$ & $56 \pm 30$ & $53 \pm 30$ \\
\hline Removal of tape & $22.6 \pm 18.9$ & $15.9 \pm 17.9$ & $27.9 \pm 27.1$ & $9 \pm 12$ & $19 \pm 21$ \\
\hline Today's puncture & $12.4 \pm 20.2$ & $9.5 \pm 20.4$ & $9.1 \pm 16.8$ & $6.6 \pm 12$ & $9.5 \pm 17$ \\
\hline \multicolumn{6}{|l|}{ All patients $\dagger$} \\
\hline Today's puncture & $16.6 \pm 24$ & $8.2 \pm 16$ & $5.1 \pm 10$ & $9.0 \pm 17$ & \\
\hline
\end{tabular}

* Comparison was done by analysis of variance for repeated measures. Comparison for two groups, by $t$ test. Values are mean \pm SD.

$\uparrow$ Mean \pm SD of all patients receiving patch and cream.

Table 5. Local reaction at site of EMLA application (\%)

\begin{tabular}{lcc}
\hline & Patch & Cream \\
\hline At site of emulsion & & \\
Itching & 5 & 5 \\
Burning & 0 & 1.5 \\
Redness & 7.5 & 2.5 \\
$\quad$ Pallor & 83.8 & 91 \\
At site of tape & & \\
$\quad$ Redness & 76.2 & 80 \\
\hline
\end{tabular}

Table 6. Correlation between recall of last puncture's pain and age, corrected time since diagnosis (cTd), and time since last puncture $(T p)$

\begin{tabular}{lcr}
\hline \multicolumn{1}{c}{ Independent variables } & $r$ & $p$ Value \\
\hline First step: variable entered age & 0.304 & 0.0001 \\
Second step: variable entered cTd & 0.347 & $<0.0001$ \\
Variable not in equation: Tp & & \\
\hline
\end{tabular}

We examined the correlation between age, cTd, time since last venipuncture, number of punctures per year, and VAS of last puncture by stepwise linear regression (Table 6). The correlation between age and VAS was the strongest $(r=0.30 ; p<0.001)$; the cTd significantly improved the correlation with VAS $(r=$ $0.34)$. No improvement in correlation was found by entering number of punctures per year and time since last puncture. Figure 1 presents the mean VAS arbitrarily stratified by two factors; diagnosis made more or less than 2 y ago and age more or less than 12 y. Figure 1 documents maximal pain in children who were younger than $12 \mathrm{y}$ of age and whose diagnosis was made less than 2 y ago ( $p<0.05$ in both cases).

Parents' and nurses' evaluations of EMLA are summarized in Tables 1 and 2, reconfirming the efficacy of EMLA.

\section{DISCUSSION}

There are potential advantages to each of the two tested methods of applying EMLA. The patch is prepared in a standardized manner, and its application may be easier for parents

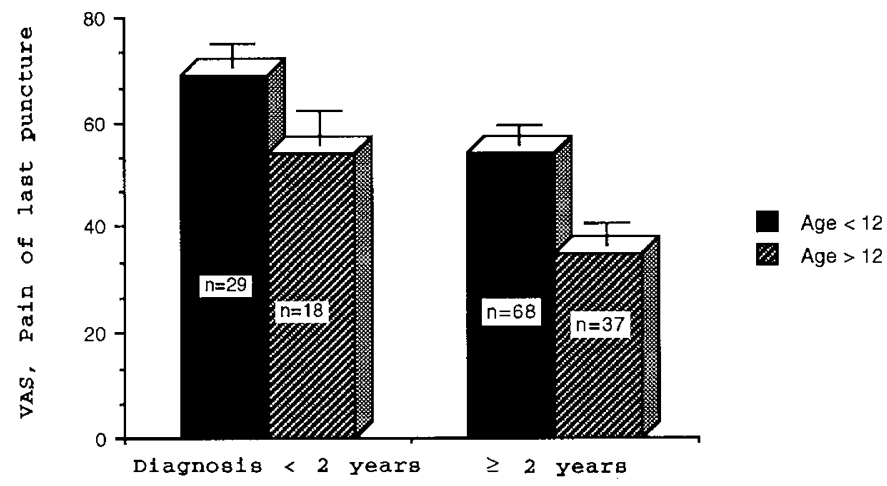

Fig. 1. Pain of the last puncture (mean VAS and SEM).

and other nonmedical individuals. Similarly, in a busy clinic setting it may be a much easier form of administration for the medical staff. Conversely, the cream may be bought and stored in large amounts, thus reducing the cost per application.

Our study reveals that although the patch and cream forms were similar in effectiveness and rate of adverse reactions, the adhesiveness of the patch was less optimal. This appears to be related to the stiffness of the foam tape; when applied to the cubital fossa or close to a joint, the flexion of the articulation may remove the patch. In comparison, Tegaderm is a much softer material and is more compatible with normal activity. A smaller patch or the use of a softer adhesive may obviate this inconvenience.

We demonstrated that children who remember having experienced severe pain during the last puncture are more likely to ask for EMLA in subsequent punctures. Previous experience of severe pain can cause a severe anxiety toward the upcoming venipuncture. McGrath (9) defined fear to be a major determinant of pain perception in children. The identification of children who fear the venipuncture most is important to help them reduce their pain by behavioral and/or pharmacologic methods, such as EMLA. Our study shows that younger children perceive more pain than older ones for an equivalent stimulus. In reality, 
however, there is evidence that young children receive less analgesia than adults in similar clinical circumstances (10).

Our study shows that children who have recently become ill have higher pain scores. Although age and the time since diagnosis are strongly correlated, these two factors contribute independently to the perception of pain by children (Fig. 1, Table 6). Children who have been sick for several years may have developed more effective coping mechanisms and a better understanding of their treatment, thus contributing to reduction in pain perception. Our study indicates that younger children who have been recently diagnosed with a chronic disease are more likely to benefit from methods that aim at helping reduce pain of venipunctures. Many other factors may play a role in pain perception, inasmuch as these two factors account for only $12 \%$ of the VAS variability. Each child therefore needs to be individually assessed, whenever possible by direct scaling techniques (11). It has been shown that cultural, familial, and psychologic factors may define the way a child is coping with pain (6). However, as stated by McGrath, research into these interrelationships is only beginning. For most hospitalized children, the venipuncture is the most vivid and feared recollection (12). Katz et al. (13) and Jay et al. (14) evaluated the characteristics of fear in children suffering from pain, pointing out that, in children undergoing injections, empathic preparation that was supportive and child-centered resulted in less adverse response. Children with cancer express their fear of needles in a variety of forms, and these are added to other forms of fear (e.g. separation and death) to create a very negative impact on their well-being (15).

The time that elapsed since the last puncture did not influence the recall of pain in our patients, suggesting that in children memory of sharp, acute pain does not fade away quickly with time. The size of the needle, which directly influences the extent of tissue damages, was found to affect pain score. Similarly, in infants, we showed that pain of venipuncture increases with the difficulty of the puncture (4).

Our data demonstrate that it is feasible to use EMLA in the setting of busy pediatric outpatient clinics. Children's assessment demonstrates the efficacy of EMLA in alleviating pain inflicted by the venipuncture, and it was also recognized by parents and nurses, most of whom do not consider the $1 \mathrm{~h}$ waiting time disruptive. In institutions where it is not economically feasible to use EMLA for venipuncture in all children, a smaller group of children who are particularly sensitive to the pain may have to be identified. Our analysis suggests that young children who have been recently diagnosed with a chronic disease may benefit the most.

Acknowledgments. The authors thank the phlebotomy team; the nurses of Hematology/Oncology Clinics, Anita Allen, B.Sc., R.N., and Valerie Chan, B.Sc.N., R.N.; the staff of Neurology Clinic, Christine Fuller, R.N.; the staff of Rheumatology Clinic, Louise Taylor, B.Sc.N., R.N., and Susan Carpenter, R.N., and the staff of Chest and Cystic Fibrosis Clinic.

\section{REFERENCES}

1. Juhlin L, Evers H 1990 EMLA: a new topical anesthetic. Adv Dermatol 5: 75-92

2. Deleted in proof

3. Hallen B, Carlson P, Uppfelt A 1985 Clinical study of a lignocaine-prilocaine cream to relieve the pain of venipuncture. Br J Anaesth 57:326-328

4. Halperin DL, Koren G, Attias D, Pellegrini E, Greenberg ML, Wyss M 1989 Topical skin anesthesia for venous, subcutaneous drug reservoir and lumbar punctures in children. Pediatrics 84:281-284

5. Robieux IC, Kumar R, Rhadakrishnan S, Koren G 1991 Assessing pain and analgesia with a lidocaine-prilocaine emulsion in infants and toddlers during venipuncture. J Pediatr 1 18:971-973

6. McGrath PJ, Unrath AM 1987 Pain in children and adolescents. Elsevier Publishing, Amsterdam, pp 47-72

7. Robieux IC, Kumar R, Rhadakrishnan S, Koren G 1990 The feasibility of using EMLA cream in pediatric outpatient clinics. Can $J$ Hosp Pharm 43: $235-236$

8. Scott J, Huskinsson EC 1976 Graphic representation of pain. Pain 2:175-184

9. McGrath PA 1990 Pain in Children: Nature, Assessment and Treatment. The Guilford Press, New York, pp 180-181

10. Schechter NL 1989 The undertreatment of pain in children: an overview. Pediatr Clin North Am 36:781-794

11. McGrath PA 1987 An assessment of children's pain: a review of behavioral, physiological and direct scaling techniques. Pain 31:147-176

12. Eland JM, Anderson JE 1977 The experience of pain in children. In: Jocox AK (ed) Pain: A Sourcebook for Nurses and Other Health Professionals. Little, Brown, Boston, pp 453-473

13. Katz ER, Varni JW, Jay SM 1984 Behavioral assessment and management of pediatric pain. Prog Behav Modif 18:163-193

14. Jay S, Elliott C, Varmi JW 1984 Acute and chronic pain in children. J Consult Clin Psychol 54:601-607

15. The Center for Attitudinal Healing 1978 There is a Rainbow Behind Every Dark Cloud. The Center for Attitudinal Healing, Berkeley, CA 